\title{
Torre de Hanoi: datos normativos y desarrollo evolutivo de la planificación
}

\author{
Alicia Díaz ${ }^{1}$, Raquel Martín², Juan E. Jiménez², Eduardo García², \\ Sergio Hernández y Cristina Rodríguez ${ }^{2}$ \\ ${ }^{1}$ Universidad de Las Palmas de Gran Canaria (España) \\ ${ }^{2}$ Universidad de La Laguna (España)
}

\begin{abstract}
El objetivo de este trabajo ha sido evaluar el desarrollo evolutivo de la planificación y obtener datos normativos de la prueba de planificación "Torre de Hanoi" en escolares de Educación Primaria (EP). Con este propósito se ha seleccionado una muestra de 1.032 alumnos/as escolarizados desde primero hasta sexto curso de EP en la Comunidad Autónoma de Canarias, con edades comprendidas entre los 6 años y 3 meses y los 12 años y 4 meses. Se analizó el número de diseños correctamente realizados por los escolares en los diferentes cursos. Los resultados encontrados ponen de manifiesto la existencia de tres momentos diferenciados en el desarrollo de la planificación, observándose un rendimiento inferior del alumnado de primer curso frente al resto de los cursos y un rendimiento más similar entre, por un lado, el alumnado de segundo, tercero y cuarto, y, por otro lado, entre el de quinto y sexto curso. Asimismo, se observa una mejora progresiva en la planificación -en función de la edad. Por último, se presenta el baremo para EP de la prueba "Torre de Hanoi”.
\end{abstract}

Palabras clave: Baremación, desarrollo evolutivo, evaluación, funciones ejecutivas, planificación, Torre de Hanoi.

Tower of Hanoi: normative data and evolutionary development of planning. The study focused on planning development in children of elementary grades and in the standardization of the test for the planning assessment "Tower of Hanoi". A sample of 1.032 was selected $1^{\text {st }}$ to $6^{\text {th }}$ grade belonging to schools in the Canary Islands with an age range between 6 years and 3 months old to 12 years and 4 months old. We analyzed the number of designs correctly in different grades. We found that there are three different moments in planning development: $1^{\text {st }}$ grade children were different from the rest of the grades; whereas children of $2^{\text {nd }}, 3^{\text {rd }}$ and $4^{\text {th }}$ grades were more similar; and children of $5^{\text {th }}$ and $6^{\text {th }}$ grades between them. We also found a gradual improvement in planning function of age. Furthermore, "Tower of Hanoi" scales are presented for children of elementary grades.

Key words: Standarization, development, assessment, executive functions, planning, Tower of Hanoi. 
La planificación es una función superior del cerebro humano que se refiere a la capacidad de pensar anticipadamente para generar acciones con el propósito de lograr un objetivo o una meta. Por tanto, se trata de una habilidad cognitiva que está relacionada con la anticipación de las consecuencias de las acciones. Concretamente, la planificación efectiva implica considerar diferentes alternativas de acción y elegir, antes de la acción, aquella más pertinente considerando las consecuencias de todas ellas. Esto supone la selección de una alternativa y la inhibición de otras (Tirapu, Céspedes y Pelegrín, 2002), lo cual implica que para que se lleve a cabo la planificación se debe contar con una adecuada capacidad de inhibición cognitiva. Ambas habilidades (planificación e inhibición cognitiva) forman parte del constructo que se ha denominado funciones ejecutivas, que es un conjunto de dominios relacionados pero distinguibles entre los que se encuentra la planificación junto con el control de los impulsos, la flexibilidad de atención y la memoria de trabajo (Welsh, Pennington y Grossier, 1991). Asimismo, la planificación requiere habilidades para representar el tiempo o el orden temporal (Benson, 1997) y un buen nivel de memoria y capacidad de sostener la atención (Soprano, 2003). En este sentido, nos encontramos que cuando tratamos de describir una de las funciones ejecutivas, en este caso la planificación, debemos de considerar la relación de esta habilidad con el resto de las funciones, ya que todas ellas actúan de forma conjunta para permitirnos funcionar adecuadamente en relación a objetivos futuros. Esta actuación conjunta no se produce de manera simultánea, sino que se ha encontrado que la inhibición cognitiva se encontraría a la base del funcionamiento ejecutivo, siendo esta habilidad mediadora de la aparición de la planificación y de la memoria de trabajo (Barkley, 1997).

El comportamiento planificado se caracteriza por no estar controlado por un estímulo inmediato, por lo que la conducta planificada resulta más flexible que la conducta no planificada caracterizándose esta última por una respuesta impulsiva guiada por el estímulo presente. En este sentido se ha observado como pacientes adultos con problemas en la flexibilidad cognitiva, presentan igualmente dificultades para planificar su vida cotidiana (Shallice, 1988).

La planificación comparte con el resto de las funciones ejecutivas la particularidad de ser un proceso que permite el mantenimiento adecuado de la información en la búsqueda de los objetivos de futuro. Todos estos procesos se han relacionado con las regiones prefrontales del cerebro (Welsh et al., 1991). Concretamente en el caso de la planificación, hay estudios que sugieren que ésta no está relacionada únicamente con una región cerebral, sino que depende de múltiples circuitos neuronales de codificación para subprocesos especializados como la memoria de trabajo, la generación de planes o la recompensa obtenida (Dehaene y Changeux, 1997). Asimismo, debido a que la planificación del comportamiento está claramente influenciada por la recompensa y dado que la corteza orbitofrontal controla el 
comportamiento voluntario, dirigido a un objetivo por los resultados de la motivación (Rolls, 2000) y la emoción (Rolls, 1999), se ha sugerido igualmente la participación de esta área en la planificación. La corteza orbitofrontal es una parte de un sistema neural integrado relacionado con una amplia red de áreas límbicas, prefrontales, sensoriales y premotoras (Barbas, 2000; Cavada, Company, Tejedor, Cruz-Rizzolo y Reinoso-Suárez, 2000; Stefanacci y Amaral, 2000). Entre la riqueza de las posibles interacciones, se ha encontrado que la interacción de la corteza orbitofrontal y la amígdala es fundamental para orientar el comportamiento sobre la base de los resultados esperados (Baxter, Parker, Lindner, Izquierdo y Murray, 2000; García, Vouimba, Baudry y Thompson, 1999; Schoenbaum, Chiba y Gallagher, 2000). Por tanto, el desarrollo de las funciones ejecutivas está íntimamente ligado a la maduración del cerebro y, especialmente, a la de la corteza prefrontal. Los cambios que se producen en esta región cerebral hacen posible el desarrollo gradual de las funciones que esta estructura sustenta (Luna et al., 2001; Tamm, Menon y Reiss, 2002). La corteza prefrontal madura en la adolescencia (Gogtay et al., 2004; O'Hare y Sowell, 2008) a diferencia de muchas otras regiones del cerebro que maduran antes (por ejemplo, las regiones involucradas en la atención, control motor, procesamiento sensorial y el lenguaje). Durante este período de desarrollo cerebral, se producen cambios progresivos y regresivos (en gran parte relacionados con la mielinización y la poda sináptica), estos se producen de forma concomitante y son impulsados en parte por las experiencias del niño, esto es así ya que el dilatado proceso de maduración de la corteza prefrontal permite que la interacción del niño con su entorno moldeen las redes neuronales que sustentan el funcionamiento ejecutivo (Sastre-Riba, Merino-Moreno y Poch-Olivé, 2007). El resultado de estos cambios es la creación de redes eficientes de las conexiones neuronales (O’Hare y Sowell, 2008). Por tanto, al igual que otros procesos ejecutivos, la capacidad de planificación y organización está relacionada neuroanatómicamente con la corteza prefrontal y sigue un proceso de desarrollo que abarca un amplio período temporal, alcanzando niveles propios del adulto alrededor de los 12 años (Luciana y Nelson, 2002).

Los investigadores que trabajan la relación entre la disfunción ejecutiva y los diversos trastornos del desarrollo han considerado de manera específica si esas poblaciones tienen dificultades específicas en la planificación y cómo estas están relacionadas con la sintomatología (Geurts, Verté, Oosterlaan, Roeyers y Sergeant, 2004; Mackinlay, Charman y Karmiloff-Smith, 2006). Así se ha encontrado en diferentes grupos clínicos, incluidas las personas con autismo o el síndrome de Asperger un deterioro en tareas que miden planificación (Ozonoff, Pennington y Rogers, 1991), Este mismo deterioro se ha puesto de manifiesto en los niños con discapacidad intelectual (Borys, Spitz y Dorans, 1982). Asimismo, en cuanto al déficit en la planificación, numerosos estudios señalan la presencia de dicho déficit en el Trastorno por Déficit de Atención con/sin Hiperactividad (TDAH) (Barkley, 2003; Nigg, Hinshaw, Carte y 
Treuting, 1998; Willcutt, Doyle, Nigg, Faraone y Pennington, 2005). Concretamente en el estudio de meta-análisis de Willcutt et al. (2005) se sugiere que los déficits que se observan más a menudo en el TDAH se dan en medidas de inhibición de la respuesta, vigilancia, memoria de trabajo y planificación. Asimismo, el déficit en planificación ha sido encontrado en escolares con TDAH no estando dicho déficit justificado por otras condiciones comórbidas como el trastorno oposicionista desafiante, trastornos de conducta o problemas lectores (Nigg et al., 1998). Por último, se ha encontrado déficit en planificación en preescolares considerados como niños de difícil manejo o control (Hughes, Dunn y White, 1998; Hughes, White, Sharpen y Dunn, 2000). Por otro lado, los pacientes con lesiones en la corteza prefrontal muestran déficits en la planificación de un objetivo dirigido a una secuencia de movimientos como en la prueba de la Torre de Hanoi (Owen, Downes, Sahakian, Polkey y Robbins, 1990; Goel y Grafman, 1995). Con la versión de la Torre de Londres, Shallice (1982) demostró que ciertos pacientes con lesiones frontales mostraban deficiencias pronunciadas en planificación que no se podría explicar en términos de otros problemas más básicos de percepción o memoria.

La capacidad de planificación puede ser evaluada mediante diferentes pruebas que permitan observar cómo se organiza el sujeto y si tiene o no presencia de un plan para resolver el problema. Entre estas pruebas destacan los test de torres como la Torre de Hanoi, Londres, o Toronto (Soprano, 2003). En el presente trabajo hemos medido la planificación mediante la administración de la Torre de Hanoi (Borys, Spitz y Dorans, 1982). En estas tareas se supone que las ejecuciones exitosas conllevan la formulación de un plan que guía la secuencia de movimientos a realizar, la retención de dicho plan, la ejecución de los movimientos, así como la supervisión y revisión del plan conforme se ejecuta la acción. Las evaluaciones con torres permiten obtener un índice cuantitativo de las habilidades de planificación, especificando el número de pasos empleados en la solución de un problema. La Torre de Hanoi, a diferencia de otras pruebas cognitivas, no se ha relacionado con un mayor cociente intelectual, aunque sí correlaciona con la edad (Welsh et al., 1991). Por otro lado, si bien la evaluación de la planificación como función ejecutiva, no tiene valor diagnóstico para el TDAH debido a que existen casos de niños con TDAH que no presentan déficits en algunas de estas funciones, además de que el déficit en el funcionamiento ejecutivo está presente en otras condiciones (v.gr., autismo), hay numerosos estudios en los que se ha evaluado el funcionamiento ejecutivo donde se evidencia que estas pruebas podrían ser utilizadas para la identificación de niños con TDAH (Barkley, 1990, 1997; Castellanos, Sonuga-Barke, Milham y Tannock, 2006; Holmes et al., 2010; Martinussen, Hayden, Hogg-Johnson y Tannock, 2005; Nigg, 2001; Willcutt, Pennington, Chhabildas, Olson y Hulslander, 2005) debido a que se encuentra de manera significativa que el grupo de niños con TDAH presentan un rendimiento inferior en estas funciones, incluyendo la planificación, que los niños de la misma edad sin TDAH. Específicamente en el estudio de Holmes et al. (2010) se obtuvo 
que usando únicamente las puntuaciones de estas pruebas, el $86 \%$ de los niños con TDAH se clasificaban correctamente. No obstante, los autores recomiendan igualmente el uso de escalas o cuestionarios de conducta en los que se recogen los síntomas del TDAH debido a que un $25 \%$ sin el trastorno fueron clasificados.

El desarrollo de instrumentos de evaluación de las funciones ejecutivas en población escolar resulta primordial debido a que existen diversos trastornos del desarrollo en los que se ve comprometido el funcionamiento ejecutivo. Por tanto, independientemente del trastorno del que se trate, la evaluación de las funciones ejecutivas resulta fundamental tanto para delimitar la gravedad de la disfunción como para planificar la intervención. Este último aspecto es importante sobre todo en la etapa escolar donde se pone de manifiesto que todas estas funciones y entre ellas la planificación resulta crucial para el desarrollo en el contexto escolar (Meltzer, 2007). Asimismo, tal y como señala Barkley (1990), hay muchos síntomas solapados entre el TDAH y otros trastornos como el trastorno de conducta o el autismo (Barkley, 1990), por lo que consideramos imprescindible que se incorporen métodos o instrumentos adicionales que sean fiables para que se pueda determinar la especificidad de estos trastornos. No obstante, a pesar de la importancia de la existencia de estos instrumentos nos encontramos que para nuestro conocimiento no existe ningún instrumento baremado para la evaluación de la planificación en el ámbito de la población infantil española. Debido a estas cuestiones hemos planteado la realización de este trabajo que tiene como principales objetivos: por un lado, estudiar el patrón de desarrollo evolutivo de esta habilidad en niños escolarizados en Educación Primaria y, por otro, realizar la baremación para la población escolar de la prueba de planificación la Torre de Hanoi.

\section{MÉTODO}

\section{Participantes}

Se seleccionó una muestra de 1.032 niños/as de edades comprendidas entre los 6 años y 3 meses y los 12 años y 4 meses. Los escolares seleccionados tenían el español como primera lengua, no presentaban daño neurológico, psiquiátrico o dificultades de aprendizaje y no formaban parte del estadillo de Educación Especial. La participación del alumnado en la administración de la prueba se hizo de manera voluntaria. En la tabla 1 se recoge la distribución de la muestra según rango de edad y sexo.

\section{Instrumentos}

Torre de Hanoi. La Torre de Hanoi (Simon, 1975) es un instrumento destinado a la evaluación de la capacidad de planificación que deriva del test de la Torre de Londres. Examina la capacidad del sujeto para resolver problemas complejos, o aprendizaje cognitivo de procedimientos. Este test exige al sujeto formular un plan para 
llegar a la solución. Este plan debe incluir un objetivo general que se descompone en diversos subobjetivos los cuales han de ser cuidadosamente ordenados. A los sujetos se les presentó una figura de madera con tres vástagos verticales de la misma longitud fijados en una base horizontal En el vástago de la izquierda estaban colocados tres cilindros de madera de tamaños progresivamente mayores ordenados de manera que el inferior era el más grande y el superior el más pequeño. En un principio, a modo de práctica, se instruyó al sujeto en mover los cilindros de uno en uno y de vástago en vástago hasta situarlos en la misma posición inicial, pero en el vástago más a la derecha. Durante el traslado de los cilindros, éstos sólo podían situarse sobre un vástago libre o sobre uno ocupado con cilindros de mayor tamaño. Nunca un cilindro podía colocarse sobre uno de tamaño menor ni fuera de la estructura. Este instrumento tiene diversas formas de aplicación, existiendo incluso versiones informatizadas, sin embargo, en el presente trabajo hemos seguido la versión aplicada en el estudio de Bishop, Aamodt, Creswell, McGurk y Skuse (2001).

\begin{tabular}{|c|c|c|}
\hline \multirow{2}{*}{$\begin{array}{l}\text { RANGO EDAD } \\
\text { (años, meses) }\end{array}$} & \multicolumn{2}{|c|}{ Sexo } \\
\hline & Varón & Mujer \\
\hline $6.3-7.4$ & 101 & 103 \\
\hline $7.5-8.4$ & 87 & 80 \\
\hline $8.5-9.4$ & 90 & 89 \\
\hline $9.5-10.4$ & 84 & 72 \\
\hline $10.5-11.4$ & 66 & 98 \\
\hline $11.5-12.4$ & 82 & 80 \\
\hline TOTAL & 510 & 522 \\
\hline
\end{tabular}

\section{Diseño y procedimiento}

Utilizamos un diseño transversal donde la variable independiente fue la Edad (6 niveles, 6.3-7.4; 7.5-8.4; 8.5-9.4; 9.5-10.4; 10.5-11.4 y 11.5-12.4 años, meses) y como variable dependiente el número de diseños correctamente realizados (número de movimientos requerido en cada nivel). La evaluación fue llevada a cabo por Psicólogas previamente entrenadas. Las evaluaciones se realizaron en un aula suficientemente iluminada y alejada de ruidos y distracciones del Centro Escolar al que asistía el alumno seleccionado. La duración de la evaluación dependía del número de ensayos realizado, ya que la prueba se dejaba de administrar cuando no se superaba un nivel de dificultad.

Las pruebas fueron administradas siguiendo la modalidad de aplicación del estudio de Bishop et al. (2001). De esta forma el examinador se sienta en frente del evaluado situando entre medio un tablero para el alumno, donde colocará los discos en la posición inicial y un tablero para el examinador, en donde colocará los discos en la posición final para que el alumno vea el modelo que ha de conseguir. Se presentan problemas con diferente nivel de dificultad en función del número de movimientos que han de ejecutar para conseguir el objetivo, habiendo problemas en los que ha de realizar $3,4,5,6,7,8$ y 9 movimientos. Se comienza siempre por el nivel inferior (3 
movimientos) y en cada nivel se presentan dos problemas diferentes (A y B) en los que se dan hasta tres intentos para resolver cada problema. Se considera que ha conseguido un nivel y, por tanto, se aumenta la dificultad, si se completa con éxito al menos uno de los problemas en dos de los tres intentos. La prueba termina cuando en ninguno de los dos problemas realiza dos intentos en el número de movimientos requerido para ese nivel. La puntuación final del alumno es equivalente al número de movimientos del último nivel superado (4 movimientos corresponde a 4 puntos), además de que se añade medio punto adicional si se han resuelto los dos problemas (A y B) de ese último nivel.

\section{RESULTADOS}

Los datos fueron analizados con la Prueba de Chi-cuadrado, ANOVA de un factor, análisis de tendencias y los correspondientes análisis post hoc todos ellos realizados mediante el paquete estadístico SPSS-PC (versión 15). Inicialmente comprobamos que la distribución de la muestra en función de la variable sexo en cada grupo de edad era similar $\left[X^{2}{ }_{(5)}=7,37 ; p=0,194\right]$. Las medias y desviaciones típicas obtenidas para cada grupo de edad en las tres pruebas administradas se muestran en la tabla 2 .

\begin{tabular}{|c|c|c|}
\hline \multirow{2}{*}{$\begin{array}{l}\text { RANGO EDAD } \\
\text { (años, meses) }\end{array}$} & \multicolumn{2}{|c|}{ Planificación (Torre de Hanoi) } \\
\hline & $X$ & $S D$ \\
\hline $6.3-7.4$ & 5.81 & 2.53 \\
\hline $7.5-8.4$ & 6.9 & 2.06 \\
\hline $8.5-9.4$ & 6.95 & 2.1 \\
\hline $9.5-10.4$ & 7.42 & 1.99 \\
\hline $10.5-11.4$ & 7.71 & 1.74 \\
\hline $11.5-12.4$ & 8.24 & 1.55 \\
\hline
\end{tabular}

\section{A. Perfil evolutivo (análisis de tendencia)}

Con el objetivo de estudiar si el nivel de rendimiento alcanzado estaba modulado por la edad llevamos a cabo un ANOVA de un factor tomando la variable edad como variable de agrupamiento y el número de diseños correctamente realizados en la prueba de la Torre de Hanoi como variable dependiente. El análisis de tendencia demostró una relación lineal entre las variables de agrupamiento para la Planificación $\left[F_{(1,866)}=117.236 ; p<.001\right]$. Se encontraron además, diferencias significativas en el rendimiento en planificación en función de la edad $\left[F_{\text {asintótica }}(5,400,985)=24.695 ; p<.001\right.$, $\omega_{\text {estimada }}^{2}=.112$ ] con un incremento del rendimiento. En la figura 1 se muestra la representación gráfica de estos resultados.

Tras este resultado llevamos a cabo contrastes a posteriori de las diferencias par a par. Los resultados mostraron que el grupo de menor edad (6.3-7.4 años, meses) tuvo un rendimiento significativamente inferior que el resto de los grupos. Por otro lado cuando contrastamos el grupo 2 (7.5-8.4 años, meses) con el resto de los grupos, 
encontramos que este grupo presenta igual rendimiento que el grupo 3 y 4 , y un rendimiento inferior que los grupos 5 y 6 . Este mismo patrón lo encontramos con el grupo 3 (8.5-9.4 años, meses). En cuanto al grupo 4 (9.5-10.4 años, meses) se obtuvo un patrón similar al de los grupos 2 y 3, pero en este caso este grupo no se diferenció del grupo 5 (10.5-11.4 años, meses) sino únicamente del grupo 6 (11.5-12.4 años, meses). Por último, los grupos 5 y 6 tienen un rendimiento similar, no diferenciándose entre ellos.

Figura 1. Perfil de Desarrollo de la Planificación

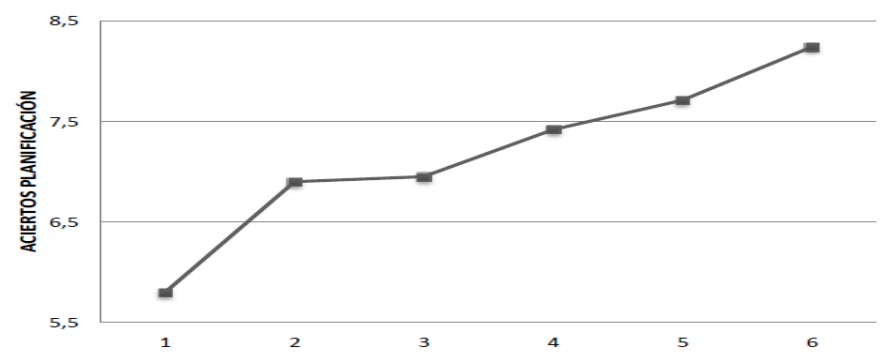

B. Estudio de baremación

Las puntuaciones medias obtenidas por cada nivel escolar (rango de edad del grupo) fueron convertidas en percentiles con el propósito de establecer una escala de los valores de las puntuaciones directas de cada grupo de edad que representan el percentil 75,50 y 25 . Los resultados encontrados pueden consultarse en la tabla 4.

Tabla 4. Baremos para planificación

\begin{tabular}{lcccccc}
\hline Planificación & Grupo 1 & Grupo 2 & Grupo 3 & Grupo 4 & Grupo 5 & Grupo 6 \\
\hline 75 & 8 & 9 & 9 & 9.5 & 9 & 9.5 \\
\hline 50 & 6 & 7 & 7 & 8 & 8.5 & 9 \\
\hline 25 & 4.5 & 5.5 & 5 & 6 & 6 \\
\hline
\end{tabular}

\section{DISCUSIÓN}

El propósito de este trabajo ha sido estudiar el desarrollo evolutivo de la planificación, mediante la administración de la prueba Torre de Hanoi que a su vez hemos baremado para la población de Educación Primaria. La medida que se utilizó para este objetivo fue la del número de diseños correctamente realizados por los escolares teniendo en cuenta el número de movimientos mínimo.

En cuanto al estudio del desarrollo evolutivo de la planificación observamos un incremento lineal en el rendimiento mostrado por los escolares en función de la edad. Los análisis posteriores nos llevan a entender posibles patrones de desarrollo de esta habilidad, observándose un momento inicial (6.3-7.4 años, meses) que se diferencia del resto de los grupos de edad. Este resultado es coincidente con otros estudios como el de 
Rosselli et al. (2004) en el que se obtuvo igualmente que el grupo de niños más pequeños (edades de 5 a 6 años) es el que se diferencia del resto de los grupos. Este resultado nos sugiere que en estas edades todavía no se ha adquirido suficientemente la capacidad de planificación, comenzándose a desarrollar hacia los 7 años de edad. Asimismo, nuestros resultados en cuanto a la mayor similitud entre las edades desde los 7 años y 5 meses hasta los 10 años y cuatro meses, coinciden con Pineda (2000) donde se expone que los niños alcanzan el desarrollo de algunas funciones ejecutivas hacia los 10 años, por lo que con anterioridad a esa edad podemos observar rendimientos más similares entre los niños de 8, 9 y 10 años. Por último, encontramos una mayor similitud entre los alumnos desde los 10 años y 5 meses hasta los 12 años y 4 meses, lo cual coincide igualmente con Pineda (2000), así como con los estudios de Rosselli, et al. (2004) y De Luca et al. (2003), donde de la misma forma, que en nuestro estudio, no se observó una mejoría significativa entre estas edades, lo cual es interpretado por lo autores como un reflejo de una relativa ignorancia de los adolescentes sobre cómo implementar o aprovechar los nuevos alcances de sus habilidades aun cuando una creciente capacidad de los circuitos neurales tiene lugar durante estas edades. Dicho de otra forma, en estas edades a pesar de que hay un desarrollo importante de los circuitos neurales, la escasa capacidad metacognitiva de los adolescentes provoca un efecto de meseta en esta tarea. Asimismo, debido a que no se obtuvo en el grupo de más edad la máxima puntuación se nos plantea igualmente la incógnita de cuándo se alcanza el control completo de esta tarea.

Por último en cuanto a los resultados del estudio de baremación, subrayamos la necesidad de realizar estudios de estandarización de pruebas de funcionamiento ejecutivo, ya que estamos de acuerdo con aquellos estudios donde se pone de manifiesto la importancia de incluir medidas de funcionamiento ejecutivo en la evaluación clínica con el objetivo de disminuir la dependencia exclusiva de los cuestionarios conductuales, así como para contribuir a la reconocida necesidad de métodos múltiples evaluaciones en la identificación de TDAH (Pineda et al., 2007). En este sentido entendemos que el diagnóstico del TDAH debe de basarse en ambos criterios (cuestionarios y pruebas de funcionamiento ejecutivo), reconociendo que si bien las pruebas de planificación no tienen valor diagnóstico por sí mismo, no es menos cierto que éstas pueden aportar información valiosa tanto en cuanto a las características del trastorno como a su evolución y pronóstico, lo que sin duda influirá en la programación de la intervención. Por otro lado, consideramos la relevancia de la utilización de recursos técnicos en la identificación y seguimiento del TDAH (Rodríguez et al., 2011). Todas estas ideas cobran especial relevancia si atendemos a los modelos actuales del TDAH que incluyen la disfunción de los procesos ejecutivos como una vía importante para entender este trastorno (Castellanos et al., 2006; Sagvolden, Johansen, Aase y Russell, 2005; SonugaBarke, 2003). Por tanto, consideramos relevante la evaluación del funcionamiento 
ejecutivo en general y de la planificación en particular cuando tratamos de identificar un posible caso de TDAH, ya que esta información nos podrá aportar un mayor entendimiento del trastorno y de las dificultades subyacentes, máxime teniendo en cuenta que tal y como señala la Asociación Americana de Pediatría (2000), los síntomas del TDAH pueden tener una gran variedad de orígenes.

\section{Agradecimientos}

Esta investigación ha sido financiada por la Agencia Canaria de Investigación, Innovación y Sociedad de la Información de la Consejería de Educación, Universidades y Sostenibilidad del Gobierno de Canarias, con ref. PI 2007/012 del que es IP el tercer autor.

\section{REFERENCIAS}

Baxter, M.G., Parker, A., Lindner, C.C., Izquierdo, A.D. y Murray, E.A. (2000). Control of response selection by reinforcer value requires interaction of amygdala and orbital prefrontal cortex. The Journal of Neuroscience, 20, 4311-4319.

Benson, J.B. (1997). The development of planning: It's about time. In S.L. Friedman y E.K. Scholnick (Eds.), The developmental psychology of planning: Why, how, and when do we plan? (pp. 43-76). Mahwah, NJ: Erlbaum.

Barbas, H. (2000). Connections underlying the synthesis of cognition memory, and emotion in primate prefrontal cortices. Brain Research Bulletin, 52, 319-330.

Barkley, R.A. (1990). Attention Deficit Hyperactivity Disorder: A handbook for diagnosis and treatment. New York: Guilford Publications.

Barkley, R.A. (1997). Behavioral inhibition, sustained attention, and executive functions: constructing a unifying theory of ADHD. Psychological Bulletin, 121(1), 65-94.

Barkley, R.A. (2003). Attention Deficit Hyperactivity Disorder. In J. Mash y R. A. Barkley (Eds.), Child Psychopathology (pp. 75-143). New York: Guilford.

Bishop, D.V., Aamodt-Leeper, G., Creswell, C., McGurk, R. y Skuse, D.H. (2001). Individual differences in cognitive planning on the Tower of Hanoi task: neuropsychological maturity or measurement error? Journal of Child Psychology and Psychiatry, 42(4), 551-556.

Borys, S.V., Spitz, H.H. y Dorans, B.A. (1982). Tower of Hanoi performance of retarded Young adults and nonretarded children as a función of solution length and goal state. Journal of Experimental Child Psychology, 10, 12-21.

Castellanos, F.X., Sonuga-Barke, E.J.S., Milham, M.P. y Tannock, R. (2006). Characterizing cognition in ADHD: Beyond executive dysfunction. Trends in Cognitive Sciences, 10, 117-123.

Cavada, C., Company, T., Tejedor, J., Cruz-Rizzolo, R.J. y Reinoso-Suárez, F. (2000). The anatomical connections of the macaque monkey orbitofrontal cortex. A review. Cereb Cortex, 10, 220-242.

Dehaene, S. y Changeux, J. (1997). A hierarchical neuronal network for planning behavior. Neurobiology, 94, 13293-13298. 
De Luca, C.R., Wood, S.J., Anderson, V.A., Buchanan, J.A., Proffitt, T.M., Mahony, K., et al. (2003). Normative data from the CANTAB. I: development of executive function over the lifespan. Journal of Clinical and Experimental Neuropsychology, 25, 242-54.

García, R., Vouimba, R.M., Baudry, M. y Thompson, R.F. (1999). The amígdala modulates prefrontal cortex activity relative to conditioned fear. Nature, 402, 294-296.

Geurts, H.M., Verté, S., Oosterlaan, J., Roeyers, H. y Sergeant, J.A. (2004). How specific are executive functioning deficits in attention deficit hyperactivity disorder and autism? Journal of Child Psychology and Psychiatry, 45, 836-854.

Goel, V. y Grafman, J. (1995). Are Frontal Lobes Implicated in Planning Functions: Re-examining the Data from the Tower of Hanoi. Neuropsychologia, 33(5), 623-442.

Gogtay, N., Giedd, J.N., Lusk, L., Hayashi K.M., Greenstein, D., Vaituzis, A.C., Nugent, T.F., Herman, D.H., Clasen, L.S., Toga, A.W., Rapoport, J.L. y Thompson, P.M. (2004). Dynamic mapping of human cortical development during childhood through early adulthood. Proceedings of the National Academy of Sciences, 101, 8174-8179.

Holmes, J., Gathercole, S., Place, M., Alloway, T., Elliott, J. y Hilton, K. (2010). The Diagnostic Utility of Executive Function Assessments in the Identification of ADHD in Children. Child and Adolescent Mental Health, 15, 37-43.

Hughes, C., Dunn, J. y White, A. (1998). Trick or treat? Uneven understanding of mind and emotion and executive dysfunction in "hard to manage" pre-schoolers. Journal of Child Psychology and Psychiatry, 39, 981-994.

Hughes, C., White, A., Sharpen, J. y Dunn, J. (2000). Antisocial, angry, and unsympathetic: "Hard-to-manage" pre-schoolers' peer problems and possible cognitive influences. Journal of Child Psychology and Psychiatry, 41, 169-179.

Luciana, M. y Nelson, C.A. (1998). The functional emergence of prefrontally-guided working memory systems in four to eight year-old children. Neuropsychologia, 36(3), 273-293.

Luna, B., Thulborn, K., Munoz, D., Merriam, E., Garver, K., Minshew, N., et al. (2001) Maturation of widely distributed brain function subserves cognitive development. Neuroimage, 13, 786-93.

Mackinlay, R., Charman, T. y Karmiloff-Smith, A. (2006). High functioning children with autism spectrum disorder: A novel test of multitasking. Brain and Cognition, 61, 14-24.

Martinussen, R., Hayden, J., Hogg-Johnson, S. y Tannock, R. (2005). A meta-analysis of working memory impairments in children attention-deficit/hyperactivity disorder. Journal of the American Academy of Child and Adolescent Psychiatry, 44, 377-384.

Meltzer, L. (2007). Executive Function in Education: From Theory to Practice. New York: Guilford.

Nigg, J.T., Hinshaw, S.P., Carte, E.T. y Treuting, J.J. (1998). Neuropsychological correlates of childhood attention deficit/hyperactivity disorder: Explainable by comorbid disruptive behavior and reading problems. Journal of Abnormal Psychology, 107, 468-480.

Nigg, J.T. (2001). Is ADHD an inhibitory disorder? Psychological Bulletin, 127, 571-598.

O'Hare, E.D. y Sowell, E.R. (2008). Imaging developmental changes in gray and white matter in the human brain. En C.A. Nelson y M. Luciana (Eds.), Handbook of Developmental Cognitive Neuroscience (pp. 23-38). Cambridge, MA: MIT Press. 
Owen, A.M., Downes, J.J., Sahakian, B.J., Polkey, C.E. y Robbins, T.W. (1990). Planning and spatial working memory following frontal lobe lesions in man. Neuropsychologia, 28(10), 1021-34.

Ozonoff, S., Pennington, B.F. y Rogers, S.J. (1991). Executive function deficits in highfunctioning autistic individuals: Relationship to theory of mind. Journal of Child Psychology and Psychiatry, 32(7), 1081-1105.

Pineda, D.A. (2000). Las funciones ejecutivas y sus trastornos. Revista de Neurología, 30, 764768.

Pineda, D.A., Palacio, I.G., Puerta, I.C., Merchan, V., Arango, C.P., Galvis, A.Y. et al. (2007). Environmental influences that affect attention deficit hyperactivity disorder: Study of a genetic isolate. European Child y Adolescent Psychiatry, 16(5), 337-346.

Rodríguez, C., González-Castro, P., Álvarez, L., Vicente, L., Núñez, J.C., González-Pienda, J.A., Álvarez, D., Bernardo, A. y Cerezo, R. (2011). Nuevas técnicas de evaluación en el Trastorno por Déficit de Atención e Hiperactividad (TDAH). European Journal of Education and Psychology, 4(1), 63-73.

Rolls, E.T. (1999). The Brain and Emotion. Oxford: Oxford University.

Rolls, E.T. (2000). The orbitofrontal cortex and reward. Cerebral Cortex, 10, 284-294.

Rosselli, M., Matute, E., Ardila, A., Botero, V.E., Tangarife, G.A., Echeverría, S.E., et al. (2004) Evaluación neuropsicológica infantil (ENI): una batería para la evaluación de niños entre 5 y 16 años de edad. Estudio normativo colombiano. Revista de Neurología, 38, 720-731.

Sagvolden, T., Johansen, E.B., Aase, H. y Russell, V.A. (2005). A dynamic developmental theory of attention-deficit/hyperactivity disorder (ADHD) predominantly hyperactive/impulsive and combined subtypes. Behavioral and Brain Sciences, 28(3), 397-419.

Sastre-Riba, S., Merino-Moreno, N. y Poch-Olivé, M.L. (2007). Formatos interactivos y funciones ejecutivas en el desarrollo temprano. Revista de Neurología, 44(2), 61-65.

Schoenbaum, G., Chiba, A.A. y Gallagher, M. (2000). Changes in functional connectivity in orbitofrontal cortex and basolateral amygdala during learning and reversal training. The Journal of Neuroscience, 20, 5179-5189.

Shallice,T. (1982). Specific impairments in planning. Philosophical Transcripts of the Royal Society of London, 298, 199-209.

Shallice,T. (1988). Specialisation within the semantic system. Cognitive Neuropsychology, 5, 135142.

Simon H.A. (1975). The functional equivalence of problem solving skills. Cognitive Psychology. 7, 268-288.

Sonuga-Barke, E.J.S. (2003). The dual pathway model of AD/HD: An elaboration of neurodevelopmental characteristics. Neuroscience Biobehavioral Review, 27, 593-604.

Soprano, A.M. (2003). Evaluación de las funciones ejecutivas en el niño. Revista de Neurología, 37(1), 44-50.

Stefanacci, L. y Amaral, D.G. (2000). Topographic organization of cortical inputs to the lateral nucleus of the macaque monkey amygdala: a retrograde tracing study. The Journal of Comparative Neurology, 421, 52-79.

Tamm, L., Menon, V. y Reiss, A. Maturation of brain function associated with response inhibition. (2002). Journal of the American Academy of Child and Adolescent Psychiatry, 41, 1231-8. 
Tirapu, J., Céspedes, J.M. y Pelegrín C. (2002). Funciones ejecutivas: necesidad de una integración conceptual. Revista de Neurología, 34, 673-685.

Welsh, M.C., Pegnnington, B.F. y Grossier, D.B. (1991). A normative-developmental study of executive functions: A window on prefrontal function in children. Developmental Neurospychology, 7, 131-149.

Willcutt, E.G., Doyle, A. E., Nigg, J. T., Faraone, S. V. y Pennington, B. F. (2005). A metaanalytic review of the executive function theory of ADHD. Biological Psychiatry, 57, 1336-1346.

Willcutt, E.G., Pennington, B.F., Chhabildas, N.A., Olson, R.K. y Hulslander, J.L. (2005). Neuropsychological analyses of comorbidity between RD and ADHD: In search of the common deficit. Developmental Neuropsychology, 27, 35-78.

Recibido: 30 de septiembre de 2011

Recepción Modificaciones: 20 de marzo de 2012

Aceptado: 22 de marzo de 2012 